\title{
Optimization and Reoptimization in Fuzzy Linear Programming problems
}

\author{
Behrouz Kheirfam ${ }^{1}$ José Luis Verdegay ${ }^{2}$ \\ ${ }^{1}$ Department of Applied Mathematics, Azarbaijan Shahid Madani University, Tabriz, I.R. Iran \\ ${ }^{2}$ Dept. of Computer Science and AI, University of Granada, Spain \\ Email: b.kheirfam@azaruniv.edu,verdegay@decsai.ugr.es
}

\begin{abstract}
Fuzzy Linear Programming models are quite frequent in practice. The dynamic nature of the real problems often requires reoptimize from the optimal solutions found, what may mean a significant consumption of time and funds. In this paper, in order to efficiently solve this problem, first the optimality conditions and the duality results for fully fuzzy linear programming problems (all parameters and variables are symmetric trapezoidal fuzzy numbers) are generalized. Then, one proposes a fuzzy dual simplex method for solving these problems without the need of converting them to conventional linear programming problems. The resulting algorithm is flexible and easy of applying. For the sake of illustration, finally, an easy example is solved.
\end{abstract}

keywords: Fuzzy linear programming, Duality theory, Dual simplex method, Trapezoidal fuzzy numbers.

\section{Introduction}

Since the addition of new constraints to a certain problem of optimization may break the feasibility of the optimal solution previously found, in the case of linear programing problems the dual simplex algorithm can be used for a rapid reoptimization without the need of finding new primal basic feasible solutions, that is, it is particularly useful for reoptimizing the solution of a certain problem after a constraint has been added or some parameters have been changed, so that the previously optimal solution may be no longer feasible. Besides this, since Linear Programming problems are almost omnipresent in all of the engineering fields, it is patent that the dual simplex algorithm is of upmost importance in a wide variety of applications and real practical problems where data, constraints or, generally speaking, parameters defining the problems may have a dynamic nature, as the algorithm facilitate to reoptimize without the need of iterating from the beginning with the consequent saving of time computation. When the data involved in the problem at hand are imprecise ones, what is quite usual in practical application, fuzzy sets are usually considered for modelling them. But in these cases, if needed, it is not possible to directly apply the dual simplex algorithm as it is not so easy like in the conventional case it is, when conventional Linear Programming problems are being considered.

As it is evident, in these circumstances there is a gap, and the main goal of this paper is to bridge this gap by presenting a dual simplex algorithm useful for the case where (trapezoidal) fuzzy numbers are involved in the problem. As it is well known, from a historical point of view, Bellman and Zadeh [2] first proposed the basic concepts of fuzzy decision making. Based on these concepts, Zimmermann [12], Tanaka and Asai [10] and Verdegay [11] formulated Fuzzy Linear Programming (FLP) problems in a fuzzy environment. Mahdavi and Nasseri [8] proposed a dual simplex algorithm directly using the primal simplex tableau for solving linear programming problems with trapezoidal fuzzy variables. Allahviranloo et al. [1] solved linear programming problems with inequality constraints in a fully fuzzy environment by converting the problems to their crisp equivalent. Lotfi et al. [5] discussed the fully fuzzy linear programming problems such that all parameters and variables are triangular fuzzy numbers. Kumar et al. [7] proposed a new algorithm to find the fuzzy optimal solution of same type of fuzzy linear programming problems. Ganesan and Veeramani [4] introduced a type of fuzzy arithmetic for symmetric trapezoidal fuzzy numbers and then proposed a primal simplex method for solving fuzzy linear programming problems without converting them to crisp linear programming problems. Nasseri and et al. [9] discussed a concept of duality for fuzzy linear programming problems introduced by Ganesan and Veeramani, and derived the weak and strong duality theorems. Recently Kheirfam and Verdegay [6] have proposed a dual simplex method for fuzzy linear programming problems as introduced by Ganesan and Veeramani, and then the sensitivity analysis for these problems was discussed.

As said above, our main aim here is the establishment of duality and complementary slackness for fully fuzzy linear programming (FFLP) problems, that is, when all the parameters are symmetric trapezoidal fuzzy numbers. From the results obtained, we develop and present for first time an orig- 
lems without to have to transform them to crisp linear programming problems, that is, not using any auxiliary intermediate model, and consequently saving computation time and providing in short a friendship and straightforward algorithm.

\section{Previous results}

In this section we present some notations, notions and results [4] that will be useful in dealing with the issues addressed in this paper.

Definition 1. A fuzzy number on $\mathbb{R}$ (real line) is said to be a symmetric trapezoidal fuzzy number if there exist real numbers $a_{1}$ and $a_{2}, a_{1} \leq a_{2}$ and $\alpha>0$, such that

$$
\mu_{\tilde{a}}(x)= \begin{cases}\frac{x}{\alpha}+\frac{\alpha-a_{1}}{\alpha}, & x \in\left[a_{1}-\alpha, a_{1}\right] ; \\ \frac{1,}{\alpha}+\frac{a_{2}+\alpha}{\alpha}, & x \in\left[a_{1}, a_{2}\right] ; \\ 0, & \text { otherwise. }\end{cases}
$$

We denote a symmetric trapezoidal fuzzy number $\tilde{a}$ by $\tilde{a}=\left(a_{1}, a_{2}, \alpha, \alpha\right)$, where $\left(a_{1}-\alpha, a_{2}+\alpha\right)$ is the support of $\tilde{a}$ and $\left[a_{1}, a_{2}\right]$ its core, and the set of all symmetric trapezoidal fuzzy numbers by $\mathcal{F}(\mathbb{R})$. $\mu_{\tilde{a}}(x)$ is called a membership function of $\tilde{a}$. The fuzzy number with the above membership function is shown in Fig. 1.

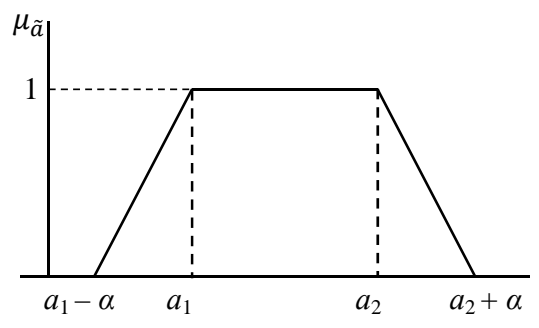

Figure 1: A symmetric trapezoidal fuzzy number.

Let $\tilde{a}=\left(a_{1}, a_{2}, \alpha, \alpha\right)$ and $\tilde{b}=\left(b_{1}, b_{2}, \beta, \beta\right)$ be two symmetric trapezoidal fuzzy numbers. The arithmetical operations on $\tilde{a}$ and $\tilde{b}$ are as follows:

1. $x>0, \quad x \in R ; \quad x \tilde{a}=\left(x a_{1}, x a_{2}, x \alpha, x \alpha\right)$,

2. $x<0, \quad x \in R ; \quad x \tilde{a}=\left(x a_{2}, x a_{1},-x \alpha,-x \alpha\right)$,

3. $\tilde{a}+\tilde{b}=\left(a_{1}+b_{1}, a_{2}+b_{2}, \alpha+\beta, \alpha+\beta\right)$.

4. $\tilde{a} \tilde{b}=\left(\left(\frac{a_{1}+a_{2}}{2}\right)\left(\frac{b_{1}+b_{2}}{2}\right)-w,\left(\frac{a_{1}+a_{2}}{2}\right)\left(\frac{b_{1}+b_{2}}{2}\right)+\right.$ $\left.w,\left|a_{2} \beta+b_{2} \alpha\right|,\left|a_{2} \beta+b_{2} \alpha\right|\right)$, where

$w=\frac{h-k}{2}, \quad k=\min \left(a_{1} b_{1}, a_{1} b_{2}, a_{2} b_{1}, a_{2} b_{2}\right)$,

$h=\max \left(a_{1} b_{1}, a_{1} b_{2}, a_{2} b_{1}, a_{2} b_{2}\right)$.

Definition 2. Let $\tilde{a}=\left(a_{1}, a_{2}, \alpha, \alpha\right)$ and $\tilde{b}=\left(b_{1}, b_{2}, \beta, \beta\right)$ be two symmetric trapezoidal fuzzy numbers. Define the relation as $\tilde{a} \preceq \tilde{b}$ if and only if either

$\frac{\left(a_{1}-\alpha\right)+\left(a_{2}+\alpha\right)}{2}<\frac{\left(b_{1}-\beta\right)+\left(b_{2}+\beta\right)}{2}$

that is $\frac{a_{1}+a_{2}}{2}<\frac{b_{1}+b_{2}}{2}$ (in this case, we can also write $\tilde{a} \prec \tilde{b})$

or

$\frac{a_{1}+a_{2}}{2}=\frac{b_{1}+b_{2}}{2}, b_{1}<a_{1}$ and $a_{2}<b_{2}$

$\frac{a_{1}+a_{2}}{2}=\frac{b_{1}+b_{2}}{2}, b_{1}=a_{1}, a_{2}=b_{2}$ and $\alpha \leq \beta$,

(in the last two cases, we can also write $\tilde{a} \approx \tilde{b}$ and say that $\tilde{a}$ and $\tilde{b}$ are equivalent).

Remark 3. Two symmetric trapezoidal fuzzy numbers $\left(a_{1}, a_{2}, \alpha, \alpha\right),\left(b_{1}, b_{2}, \beta, \beta\right)$ are equivalent if and only if

$$
\frac{a_{1}+a_{2}}{2}=\frac{b_{1}+b_{2}}{2} .
$$

In this case, we simply write $\left(a_{1}, a_{2}, \alpha, \alpha\right) \approx$ $\left(b_{1}, b_{2}, \beta, \beta\right)$ and it is to be noted that $a_{1}$ need not be equal to $b_{1}$ or $a_{2}$ need not be equal to $b_{2}$, but $\left(a_{1}, a_{2}, \alpha, \alpha\right)-\left(b_{1}, b_{2}, \beta, \beta\right) \approx(-h, h, \alpha+\beta, \alpha+\beta)$, where $h=\left(b_{2}-a_{1}\right) \geq 0$.

Remark 4. For any symmetric trapezoidal fuzzy number $\tilde{a}$, we define $\tilde{a} \succeq \tilde{0}$ if there exist $c \geq 0$ and $\delta \geq 0$ such that $\tilde{a} \succeq(-c, c, \delta, \delta)$. We also denote $(-c, c, \delta, \delta)$ by $\tilde{0}$.

In the sequel, we propose a symmetric trapezoidal fuzzy number and two new types of fuzzy arithmetic operations.

Definition 5. For any symmetric trapezoidal fuzzy number $\tilde{a}$, we define $\tilde{a} \succeq \tilde{1}$ if there exist $c \geq 0$ and $\delta \geq 0$ such that $\tilde{a} \succeq(-c+1, c+1, \delta, \delta)$. We also denote $(-c+1, c+1, \delta, \delta)$ by $\tilde{1}$. Note that $\tilde{1}$ is equivalent to $(1,1,0,0)$.

Definition 6. Let $\tilde{a}=\left(a_{1}, a_{2}, \alpha, \alpha\right)$ and $\tilde{b}=$ $\left(b_{1}, b_{2}, \beta, \beta\right)$ be symmetric trapezoidal fuzzy number and non-zero symmetric trapezoidal fuzzy number, respectively. Now, we define two types of arithmetical operations as follows:

1. Inverse: $\frac{1}{\tilde{b}}=\tilde{b}^{-1} \approx\left(\frac{2}{b_{1}+b_{2}}-w, \frac{2}{b_{1}+b_{2}}+w, \beta, \beta\right)$ where $w=\frac{h-k}{2}, h=\max _{j=1,2}\left\{\frac{1}{\bar{b}_{j}}\right\}, k=\min _{j=1,2}\left\{\frac{1}{\bar{b}_{j}}\right\}$ and $\frac{1}{\bar{b}_{j}}=\left\{\begin{array}{l}\frac{1}{b_{j}}, \quad b_{j} \neq 0 \\ 0, \quad b_{j}=0 .\end{array}\right.$

2. Division: $\frac{\tilde{a}}{\tilde{b}} \approx\left(\frac{a_{1}+a_{2}}{b_{1}+b_{2}}-w, \frac{a_{1}+a_{2}}{b_{1}+b_{2}}+\right.$ $\left.w,\left|a_{2} \beta+\frac{\alpha}{\bar{b}_{2}}\right|,\left|a_{2} \beta+\frac{\alpha}{\bar{b}_{2}}\right|\right)$ where $w=\frac{h-k}{2}, h=$ $\max _{i, j=1,2}\left\{\frac{a_{i}}{\bar{b}_{j}}\right\}, k=\min _{i, j=1,2}\left\{\frac{a_{i}}{\bar{b}_{j}}\right\}$

Lemma 7. [3] Suppose $\tilde{a}, \tilde{b}, \tilde{c} \in \mathcal{F}(\mathbb{R})$ such that $\tilde{a} \preceq \tilde{b}$. We have

1. If $\tilde{c} \succeq \tilde{0}$, then $\tilde{c} \tilde{a} \preceq \tilde{c} \tilde{b}$

2. If $\tilde{c} \preceq \tilde{0}$, then $\tilde{c} \tilde{a} \succeq \tilde{c} \tilde{b}$ 
Lemma 8. [4] For any symmetric trapezoidal fuzzy number $\tilde{a}, \tilde{b}$ and $\tilde{c}$, we have

$$
\tilde{c}(\tilde{a}+\tilde{b}) \approx \tilde{c} \tilde{a}+\tilde{c} \tilde{b}, \quad \tilde{c}(\tilde{a}-\tilde{b}) \approx \tilde{c} \tilde{a}-\tilde{c} \tilde{b}
$$

Lemma 9. [3] Suppose $\tilde{a}, \tilde{b}, \tilde{c} \in \mathcal{F}(\mathbb{R})$. We have

1. $\tilde{a} \tilde{b} \succeq \tilde{0}$, if and only if $\tilde{a} \succeq \tilde{0}$ and $\tilde{b} \succeq \tilde{0}$, or $\tilde{a} \preceq \tilde{0}$ and $\tilde{b} \preceq \tilde{0}$.

2. $\tilde{a} \tilde{b} \preceq \tilde{0}$, if and only if $\tilde{a} \succeq \tilde{0}$ and $\tilde{b} \preceq \tilde{0}$, or $\tilde{a} \preceq \tilde{0}$ and $\tilde{b} \succeq \tilde{0}$.

Lemma 10. Let $\tilde{a} \in \mathcal{F}(\mathbb{R})$ and $\tilde{a} \nsucceq \tilde{0}$. Then $\frac{\tilde{a}}{\tilde{a}} \approx \tilde{1}$

Proof. Let $\tilde{a} \approx\left(a_{1}, a_{2}, \alpha, \alpha\right)$. We have

$$
\begin{aligned}
\frac{\tilde{a}}{\tilde{a}} & \approx\left(\frac{a_{1}+a_{2}}{a_{1}+a_{2}}-w, \frac{a_{1}+a_{2}}{a_{1}+a_{2}}+w,\left|a_{2} \beta+\frac{\alpha}{\bar{a}_{2}}\right|,\left|a_{2} \beta+\frac{\alpha}{\bar{a}_{2}}\right|\right) \\
& \approx\left(1-w, 1+w,\left|a_{2} \beta+\frac{\alpha}{\bar{a}_{2}}\right|,\left|a_{2} \beta+\frac{\alpha}{\bar{a}_{2}}\right|\right) \approx \tilde{1} .
\end{aligned}
$$

Definition 11. An $n$-tuple $\tilde{a}=\left(\tilde{a}_{1}, \tilde{a}_{2}, \ldots, \tilde{a}_{n}\right)$, which $\tilde{a}_{i}$ is a symmetric trapezoidal fuzzy number for every $i=1,2, \ldots, n$, is called a symmetric trapezoidal fuzzy vector. The set of all symmetric trapezoidal fuzzy vectors is denoted by $\mathcal{F}(\mathbb{R})^{n}$.

Definition 12. Fuzzy vectors $\tilde{a}^{(1)}, \tilde{a}^{(2)}, \ldots, \tilde{a}^{(n)}$ are called fuzzy linearly dependent if there is at least one a fuzzy number $\tilde{\lambda}_{i} \nsucceq 0 \tilde{0}, i=1,2, \ldots, n$ such that $\sum_{i=1}^{n} \tilde{\lambda}_{i} \tilde{a}^{(i)} \approx \tilde{0}$. In other case, we say that these vectors are fuzzy linearly independent.

Definition 13. A symmetric trapezoidal fuzzy matrix is any rectangular array of symmetric trapezoidal fuzzy numbers. We denote by $\tilde{A}=\left[\tilde{a}_{i j}\right]_{m \times n}$, where $\tilde{a}_{i j} \in \mathcal{F}(\mathbb{R}), i=1,2, \ldots, m, j=1,2, \ldots, n$. The set of all symmetric trapezoidal fuzzy matrices is denoted by $\mathcal{F}(\mathbb{R})^{m \times n}$.

Definition 14. Let $\tilde{A} \in \mathcal{F}(\mathbb{R})^{m \times n}$, and $\tilde{a}_{j} \in$ $\mathcal{F}(\mathbb{R})^{n}$ denotes its $j$ th column, for $j=1,2, \ldots, n$. The rank of matrix $\tilde{A}$ is the largest number of fuzzy linearly independent vectors $\tilde{a}_{j}$.

Corresponding to any square matrix $\tilde{A} \in$ $\mathcal{F}(\mathbb{R})^{n \times n}$, there is a symmetric trapezoidal fuzzy number which is called the determinant of $\tilde{A}$ and is denoted by $|\tilde{A}|$. For any values of indices $i$ and $j$, the $i j$ th minor of $\tilde{A}$, denoted by $\tilde{A}_{i j}$, is the $(n-1) \times(n-1)$ sub-matrix of $\tilde{A}$ obtained by deleting the $i$ th row and the $j$ th column of $\tilde{A}$. We compute $|\tilde{A}|$ as follows:

1. For $n=1,|\tilde{A}|=\tilde{a}_{11}$.

2. For $n=2,|\tilde{A}|=\tilde{a}_{11} \tilde{a}_{22}-\tilde{a}_{21} \tilde{a}_{12}$.

3. For $n>2,|\tilde{A}|=\sum_{j=1}^{n}(-1)^{i+j} \tilde{a}_{i j}\left|\tilde{A}_{i j}\right|$,

for any value of index $i=1,2, \ldots, n$.

For a given square matrix $\tilde{A} \in \mathcal{F}(\mathbb{R})^{n \times n}$, we say that matrix $\tilde{B} \in \mathcal{F}(\mathbb{R})^{n \times n}$ is inverse of $\tilde{A}$, denoted by $\tilde{B} \approx \tilde{A}^{-1}$, if $\tilde{A} \tilde{B} \approx \tilde{B} \tilde{A} \approx \tilde{I}_{n}$, where $\tilde{I}_{n}$ is an identity matrix. A matrix $\tilde{A} \in \mathcal{F}(\mathbb{R})^{n \times n}$ is said to be singular fuzzy matrix if $|\tilde{A}| \approx \tilde{0}$. In the other case, matrix $\tilde{A}$ is called non-singular fuzzy matrix. For a non-singular fuzzy matrix $\tilde{A}$, the inverse fuzzy matrix $\tilde{A}^{-1}$ is calculated by

$$
\tilde{A}^{-1} \approx \frac{(1,1,0,0)}{|\tilde{A}|}\left[(-1)^{i+j}\left|\tilde{A}_{i j}\right|\right]_{n \times n},
$$

where $\tilde{A}_{i j}$ is the $i j$ th minor of the matrix $\tilde{A}$.

\section{Fully fuzzy linear programming}

A fully fuzzy linear programming (FFLP) problem with symmetric trapezoidal fuzzy numbers is defined as:

$$
\begin{aligned}
\max & \tilde{z} \approx \tilde{c} \tilde{x} \\
\text { s.t. } & \tilde{A} \tilde{x} \preceq \tilde{b} \\
& \tilde{x} \succeq \tilde{0},
\end{aligned}
$$

where $\tilde{b} \in \mathcal{F}(\mathbb{R})^{m}, \tilde{A} \in \mathcal{F}(\mathbb{R})^{m \times n}, \tilde{c}^{T} \in \mathcal{F}(\mathbb{R})^{n}$ are given and $\tilde{x} \in \mathcal{F}(\mathbb{R})^{n}$ is to be determined.

Definition 15. A fuzzy vector $\tilde{x}=\left(\tilde{x}_{1}, \ldots, \tilde{x}_{n}\right)^{T} \in$ $(\mathcal{F}(\mathbb{R}))^{n}$, where each $\tilde{x}_{i} \in \mathcal{F}(\mathbb{R})$, is called a fuzzy feasible solution to (FFLP) if $\tilde{x} \succeq \tilde{0}$ satisfies the constraints $\tilde{A} \tilde{x} \preceq \tilde{b}$.

Definition 16. Let $S$ be the set of all fuzzy feasible solutions of (FFLP). A fuzzy feasible solution $\tilde{x}_{*} \in$ $S$ is said to be a fuzzy optimal solution to (FFLP) if $\tilde{c} \tilde{x} \preceq \tilde{c} \tilde{x}_{*}$ for all $\tilde{x} \in S$.

\subsection{Fuzzy basic feasible solution}

We consider the following standard FFLP problem:

$$
\begin{array}{cl}
\max & \tilde{z} \approx \tilde{c} \tilde{x} \\
\text { s.t. } & \tilde{A} \tilde{x} \approx \tilde{b} \\
& \tilde{x} \succeq \tilde{0},
\end{array}
$$

where the parameters of the problem are as defined in (FFLP). Let $\tilde{A}=\left[\tilde{a}_{i j}\right]_{m \times n}$ and $\operatorname{rank}(\tilde{A})=m$. Partition $\tilde{A}$ as $\left[\begin{array}{cc}\tilde{B} & \tilde{N}\end{array}\right]$ where $\tilde{B}, m \times m$, is nonsingular fuzzy matrix (it means that $|\tilde{B}| \nsucceq \tilde{0}$ ). Let $\tilde{y}_{j}$ be the fuzzy solution to $\tilde{B} \tilde{y}_{j} \approx \tilde{a}_{j}$, where $\tilde{a}_{j}$ is the $j$ th column of the coefficients matrix $\tilde{A}$. It is apparent that the fuzzy basic solution

$$
\tilde{x}_{B} \approx\left(\tilde{x}_{B_{1}}, \ldots, \tilde{x}_{B_{m}}\right) \approx \tilde{B}^{-1} \tilde{b}, \tilde{x}_{N} \approx \tilde{0},
$$

is a fuzzy solution of $\tilde{A} \tilde{x} \approx \tilde{b}$. We call $\tilde{x} \approx$ $\left(\tilde{x}_{B}^{T}, \tilde{x}_{N}^{T}\right)^{T}$, a fuzzy basic solution corresponding to basis $\tilde{B}$. If $\tilde{x}_{B} \succeq \tilde{0}$, then the fuzzy basic solution is feasible and the corresponding fuzzy objective value is $\tilde{z} \approx \tilde{c}_{B} \tilde{x}_{B}$, where $\tilde{c}_{B} \approx\left(\tilde{c}_{B_{1}}, \ldots, \tilde{c}_{B_{m}}\right)$. Now, corresponding to every $j, 1 \leq j \leq n$, define

$$
\tilde{z}_{j} \approx \tilde{c}_{B} \tilde{y}_{j} \approx \tilde{c}_{B} \tilde{B}^{-1} \tilde{a}_{j} .
$$

Theorem 17. (Optimality conditions) Assume the FFLP problem is non-degenerate and $\tilde{B}$ is a fuzzy feasible basis. A fuzzy basic feasible solution $\tilde{x}_{B} \approx$ $\tilde{B}^{-1} \tilde{b} \succeq \tilde{0}, \tilde{x}_{N} \approx \tilde{0}$ is optimal to (1) if and only if $\tilde{z}_{j} \approx \tilde{c}_{B} \tilde{B}^{-1} \tilde{a}_{j} \succeq \tilde{c}_{j}$ for all $j, 1 \leq j \leq n$. 
Proof. Suppose $\tilde{x}_{*} \approx\left(\tilde{x}_{B}^{T}, \tilde{x}_{N}^{T}\right)^{T}$ is a fuzzy basic feasible solution to $(1)$ where $\tilde{x}_{B} \approx \tilde{B}^{-1} \tilde{b}, \tilde{x}_{N} \approx \tilde{0}$. Then the corresponding fuzzy objective value is:

$$
\tilde{z}_{*} \approx \tilde{c} \tilde{x}_{*} \approx \tilde{c}_{B} \tilde{x}_{B} \approx \tilde{c}_{B} \tilde{B}^{-1} \tilde{b} .
$$

On the other hand, for any fuzzy basic feasible solution $\tilde{x}$ to $(1)$, we have

$$
\tilde{x}_{B} \approx \tilde{B}^{-1} \tilde{b}-\tilde{B}^{-1} \tilde{N} \tilde{x}_{N} .
$$

Then

$$
\begin{aligned}
\tilde{z} & \approx \tilde{c} \tilde{x} \approx \tilde{c}_{B} \tilde{x}_{B}+\tilde{c}_{N} \tilde{x}_{N} \\
& \approx \tilde{c}_{B} \tilde{B}^{-1} \tilde{b}+\left(\tilde{c}_{N}-\tilde{c}_{B} \tilde{B}^{-1} \tilde{N}\right) \tilde{x}_{N} \\
& \approx \tilde{c}_{B} \tilde{B}^{-1} \tilde{b}+\sum_{j \in N}\left(\tilde{c}_{j}-\tilde{c}_{B} \tilde{B}^{-1} \tilde{a}_{j}\right) \tilde{x}_{j} \\
& \approx \tilde{c}_{B} \tilde{B}^{-1} \tilde{b}+\sum_{j \in N}\left(\tilde{c}_{j}-\tilde{z}_{j}\right) \tilde{x}_{j}
\end{aligned}
$$

Hence, using (4) and (5), we have

$$
\tilde{z} \approx \tilde{z}_{*}+\sum_{j \in N}\left(\tilde{c}_{j}-\tilde{z}_{j}\right) \tilde{x}_{j} .
$$

Now, if for $j \in N$ we have $\tilde{c}_{j}-\tilde{z}_{j} \preceq \tilde{0}$, then from feasibility of $\tilde{x}$ we have $\left(\tilde{c}_{j}-\tilde{z}_{j}\right) \tilde{x} \preceq \tilde{0}$, and then we obtain $\sum_{j \in N}\left(\tilde{c}_{j}-\tilde{z}_{j}\right) \tilde{x}_{j} \preceq \tilde{0}$. Therefore, it follows that $\tilde{z} \preceq \tilde{z}_{*}$, and so $\tilde{x}_{*}$ is optimal.

Remark 18. If $\tilde{A}$ and $\tilde{c}$ are crisp numbers, then the above optimality condition matches the optimality condition in [8]. Moreover, if the data and variables are crisp numbers, then the above theorem is consistent with the optimality condition for crisp linear programming.

In the next section, we develop the duality results

\section{Duality}

We consider the (FFLP) problem. We define the dual problem of (FFLP) as follows:

$$
\begin{array}{cc}
\min & \tilde{u} \approx \tilde{w} \tilde{b} \\
\text { s.t. } & \tilde{w} \tilde{A} \succeq \tilde{c} \\
& \tilde{w} \succeq \tilde{0},
\end{array}
$$

where $\tilde{w}^{T} \in \mathcal{F}(\mathbb{R})^{m}$.

Theorem 19. (The weak duality properties) If $\tilde{x}$ and $\tilde{w}$ are fuzzy feasible solutions to (FFLP) and (DFFLP) problems, respectively, then $\tilde{w} \tilde{b} \succeq \tilde{c} \tilde{x}$.

Proof. Multiplying $\tilde{A} \tilde{x} \preceq \tilde{b}$ on the left by $\tilde{w} \succeq \tilde{0}$ and $\tilde{w} \tilde{A} \succeq \tilde{c}$ on the right by $\tilde{x} \succeq \tilde{0}$ and using Lemma 7 , we get $\tilde{c} \tilde{x} \preceq \tilde{w} \tilde{A} \tilde{x} \preceq \tilde{w} \tilde{b}$.

Corollary 20. If $\tilde{x}$ and $\tilde{w}$ are fuzzy feasible solutions to (FFLP) and (DFFLP) problems, respectively, and $\tilde{w} \tilde{b} \approx \tilde{c} \tilde{x}$, then $\tilde{x}$ and $\tilde{w}$ are fuzzy optimal solutions to their respective problems.

Proof. It is straightforward, using Theorem 19.
Definition 21. We say the (FFLP) problem (or the (DFFLP) problem) is unbounded if fuzzy feasible solutions exist that the fuzzy objective value is increased (or decreased) indefinitely.

The following result relates unboundedness of one problem to infeasibility of the other.

Corollary 22. If any one of the (FFLP) or (DF$F L P)$ problem is unbounded, then the other problem has no fuzzy feasible solution.

Proof. Suppose that the (FFLP) is unbounded and that the (DFFLP) problem has a fuzzy feasible solution $\tilde{w}$. By Theorem 19, we have $\tilde{c} \tilde{x} \preceq \tilde{w} \tilde{b}$ for every fuzzy feasible solution $\tilde{x}$. This shows that $\tilde{w} \tilde{b}$ is an upper bound of $\tilde{c} \tilde{x}$. This is impossible and the dual can not have a fuzzy feasible solution. Thus, the proof is complete.

Theorem 23. (Strong duality) If any one of the (FFLP) or (DFFLP) problem has a fuzzy optimal solution, then both problems have fuzzy optimal solutions and the fuzzy optimal objective values are equal.

Proof. Assume that the (FFLP) problem has a fuzzy optimal solution. Let $\tilde{v} \succeq \tilde{0}$ be the fuzzy slack variables for the constraints $\tilde{A} \tilde{x} \preceq \tilde{b}$. Then, we have

$$
\begin{array}{cc}
\max & \tilde{z} \approx \tilde{c} \tilde{x}+\tilde{0} \tilde{v} \\
\text { s.t. } & \tilde{A} \tilde{x}+\tilde{v} \approx \tilde{b} \\
& \tilde{x}, \tilde{v} \succeq \tilde{0} .
\end{array}
$$

Assume $\tilde{B}$ is the optimal basis matrix and $\tilde{x}_{*} \approx$ $\left(\tilde{x}_{B}^{T}, \tilde{0}\right)^{T} \approx\left(\left(\tilde{B}^{-1} \tilde{b}\right)^{T}, \tilde{0}\right)^{T}$ is the fuzzy basic optimal solution corresponding to the (FFLP) problem. From Theorem 17 we have, the corresponding to $\tilde{x}_{j}, j=1,2, \ldots, n$,

$$
\tilde{z}_{j}-\tilde{c}_{j} \approx \tilde{c}_{B} \tilde{B}^{-1} \tilde{a}_{j}-\tilde{c}_{j} \succeq \tilde{0},
$$

and the corresponding to $\tilde{v}_{i}, i=1,2, \ldots, m$,

$$
\tilde{c}_{B} \tilde{B}^{-1} \tilde{e}_{i} \succeq \tilde{0}
$$

where $\tilde{e}_{i} \approx(\tilde{0}, \ldots, \tilde{1}, \ldots, \tilde{0})^{T}$. Now, let $\tilde{w}_{*} \approx$ $\tilde{c}_{B} \tilde{B}^{-1}$. Using the above inequalities, we can write,

$$
\begin{gathered}
\tilde{w}_{*} \tilde{A} \succeq \tilde{c}, \\
\tilde{w}_{*} \succeq \tilde{0} .
\end{gathered}
$$

Thus, $\tilde{w}_{*}$ is a fuzzy feasible solution to the (DFFLP) problem and

$$
\tilde{w}_{*} \tilde{b} \approx \tilde{c}_{B} \tilde{B}^{-1} \tilde{b} \approx \tilde{c}_{B} \tilde{x}_{B} \approx \tilde{c} \tilde{x}_{*} .
$$

Therefore, the result follows immediately from Corollary 20.

Theorem 24. (Complementary slackness) Let $\tilde{x}_{*}$ and $\tilde{w}_{*}$ be any fuzzy feasible solutions to (FFLP) and (DFFLP) problems, respectively. Then $\tilde{x}_{*}$ and $\tilde{w}_{*}$ are fuzzy optimal solutions if and only if

$$
\left(\tilde{w}_{*} \tilde{A}-\tilde{c}\right) \tilde{x}_{*} \approx \tilde{0}, \quad \tilde{w}_{*}\left(\tilde{b}-\tilde{A} \tilde{x}_{*}\right) \approx \tilde{0} .
$$


Proof. Suppose that $\tilde{x}_{*}$ and $\tilde{w}_{*}$ are fuzzy feasible solutions to (FFLP) and (DFFLP) problems, respectively. By Theorem 23, we have

$$
\begin{aligned}
\tilde{0} \approx \tilde{c} \tilde{x}_{*}-\tilde{w}_{*} \tilde{b} & \approx\left(\tilde{c} \tilde{x}_{*}-\tilde{w}_{*} \tilde{A} \tilde{x}_{*}\right)+\left(\tilde{w}_{*} \tilde{A} \tilde{x}_{*}-\tilde{w}_{*} \tilde{b}\right) \\
& \approx\left(\tilde{c}-\tilde{w}_{*} \tilde{A}\right) \tilde{x}_{*}+\tilde{w}_{*}\left(\tilde{A} \tilde{x}_{*}-\tilde{b}\right) .
\end{aligned}
$$

On the other hand, we have $\left(\tilde{c}-\tilde{w}_{*} \tilde{A}\right) \tilde{x}_{*} \preceq \tilde{0}$ and $\tilde{w}_{*}\left(\tilde{A} \tilde{x}_{*}-\tilde{b}\right) \preceq \tilde{0}$. Therefore, we obtain

$$
\left(\tilde{c}-\tilde{w}_{*} \tilde{A}\right) \tilde{x}_{*} \approx \tilde{0}, \text { and } \tilde{w}_{*}\left(\tilde{A} \tilde{x}_{*}-\tilde{b}\right) \approx \tilde{0} .
$$

The converse of the theorem follows from the fact that $\left(\tilde{c}-\tilde{w}_{*} \tilde{A}\right) \tilde{x}_{*} \approx \tilde{0}$, and $\tilde{w}_{*}\left(\tilde{A} \tilde{x}_{*}-\tilde{b}\right) \approx \tilde{0}$ imply that $\tilde{c} \tilde{x}_{*} \approx \tilde{w}_{*} \tilde{b}$. Therefore, optimality of $\tilde{x}_{*}$ and $\tilde{w}_{*}$ follows from Corollary 20.

Remark 25. If $\tilde{A}$ and $\tilde{c}$ are crisp numbers, then the above results reduce to the duality theorems in [8]. Moreover, if the all data and variables are crisp numbers, then these results are consistent with the results of linear programming.

\section{Dual simplex method}

Consider the (FFLP) problem. We may rewrite (FFLP) as follows:

$$
\begin{array}{cc}
\max & \tilde{z} \approx \tilde{c} \tilde{x}+\tilde{0} \tilde{v} \\
\text { s.t. } & \tilde{A} \tilde{x}+\tilde{v} \approx \tilde{b} \\
\tilde{x}, \tilde{v} \succeq \tilde{0},
\end{array}
$$

where $\tilde{v} \approx\left(\tilde{v}_{1}, \ldots, \tilde{v}_{m}\right)^{T}$.

Define $\tilde{x} \in \mathcal{F}(\mathbb{R})^{n+m}$ and $\tilde{c} \in \mathcal{F}(\mathbb{R})^{n+m}$ as

$$
\begin{gathered}
\tilde{x}_{j}= \begin{cases}\tilde{x}_{j}, & j=1,2, \ldots, n, \\
\tilde{v}_{j-n}, & j=n+1, \ldots, n+m,\end{cases} \\
\tilde{c}_{j}= \begin{cases}\tilde{c}_{j}, & j=1,2, \ldots, n, \\
\tilde{0}, & j=n+1, \ldots, n+m .\end{cases}
\end{gathered}
$$

Suppose that a fuzzy basic solution for (FFLP) is given by $\tilde{x}_{B} \approx \tilde{B}^{-1} \tilde{b}$ and $\tilde{x}_{N} \approx \tilde{0}$, with the basis matrix $\tilde{B}$. Now let $\tilde{z}_{j} \approx \tilde{c}_{B} \tilde{B}^{-1} \tilde{a}_{j}, \tilde{y}_{0} \approx \tilde{B}^{-1} \tilde{b}$, where $\tilde{c}_{B} \approx\left(\tilde{c}_{B_{1}}, \ldots, \tilde{c}_{B_{m}}\right)$ and $\tilde{a}_{j}$ is the $j$ th column of the coefficient matrix $\tilde{A}$. Consider Table 1 , where $\tilde{x}_{B_{r}}$ is the $r$ th fuzzy basic variable and $\tilde{y}_{j} \approx \tilde{B}^{-1} \tilde{a}_{j}$.

Suppose that for $j=1, \ldots, n+m$, we have

$$
\tilde{z}_{j}-\tilde{c}_{j} \approx \tilde{c}_{B} \tilde{B}^{-1} \tilde{a}_{j}-\tilde{c}_{j} \succeq \tilde{0},
$$

that is, the optimality condition of the (FFLP) at $\tilde{x}$ holds true. we define $\tilde{w} \approx \tilde{c}_{B} \tilde{B}^{-1}$, where $\tilde{w} \approx$ $\left(\tilde{w}_{1}, \ldots, \tilde{w}_{m}\right)$. In this way, from (8), we have

$$
\tilde{w} \tilde{A} \succeq \tilde{c}, \tilde{w} \succeq \tilde{0}
$$

that is, $\tilde{w}$ is a dual fuzzy feasible solution. If $\tilde{y}_{0 r} \succeq \tilde{0}$, for all $r=1, \ldots, m$, then we can obtain a fuzzy feasible solution for the (FFLP) problem. Moreover, we will have

$$
\tilde{c} \tilde{x} \approx \tilde{c}_{B} \tilde{x}_{B} \approx \tilde{c}_{B} \tilde{B}^{-1} \tilde{b} \approx \tilde{w} \tilde{b},
$$

and thus, by Corollary 20, establish the optimality of $\tilde{x}$ and $\tilde{w}$ for the (FFLP) and (DFFLP), respectively. Therefore, we have the following result.
Corollary 26. The optimality criteria $\tilde{z}_{j}-\tilde{c}_{j} \succeq \tilde{0}$ for all $j$, for the (FFLP) problem is equivalent to the feasibility condition for the (DFFLP) problem. If, in addition, $\tilde{x}$ corresponding to a basis $\tilde{B}$ is primal fuzzy feasible then $\tilde{x}$ is optimal for the (FFLP) problem and $\tilde{w} \approx \tilde{c}_{B} \tilde{B}^{-1}$ is optimal to the (DFFLP) problem.

Now, assume that the (DFFLP) problem is feasible and $\tilde{x}$, corresponding to a basis $\tilde{B}$, is dual feasible but primal infeasible. That is, we have

$$
\tilde{z}_{j}-\tilde{c}_{j} \succeq \tilde{0}, \forall j,
$$

and there exists at least one $r$ such that $\tilde{y}_{0 r} \prec \tilde{0}$. Thus, according to duality theory, the (FFLP) problem can be either infeasible (in which case, the (FLD) problem is unbounded), or it has an optimal solution. Next we will show how to work on row $r$ of the above tableau corresponding to basis $\tilde{B}$, as the pivoting row, and either (1) detect the infeasibility of the (FFLP) problem (or unboundedness of the (DFFLP) problem), or (2) find a column $\ell$, as a pivoting column, to pivot on $\tilde{y}_{r \ell}$ and obtain a new dual feasible tableau with a non-increasing primal objective value. We explain these cases below.

Theorem 27. If in a dual feasible simplex tableau an $r$ exists such that $\tilde{y}_{0 r} \prec \tilde{0}$ and $\tilde{y}_{r j} \succeq \tilde{0}$, for all $j$, then the (FFLP) problem is infeasible.

Proof. Suppose that Tableau 1 is a dual feasible tableau, and an $r$ exists such that $\tilde{y}_{0 r} \prec \tilde{0}$ and $\tilde{y}_{r j} \succeq \tilde{0}$ for all $j$. Corresponding to the $r$ th row of the tableau, we have

$$
\tilde{x}_{B_{r}}+\sum_{j \in N} \tilde{y}_{r j} \tilde{x}_{j} \approx \tilde{y}_{0 r} .
$$

Since, by assumption, $\tilde{y}_{r j} \succeq \tilde{0}, j \in N$ and $\tilde{x}_{j} \succeq \tilde{0}$, then $\tilde{x}_{B_{r}}+\sum_{j \in N} \tilde{y}_{r j} \tilde{x}_{j} \succeq \tilde{0}$ for any fuzzy basic feasible solution. However, $\tilde{y}_{0 r} \prec \tilde{0}$ and this shows that the (FFLP) problem is infeasible.

Theorem 28. If in a dual feasible simplex tableau, an $r$ exists such that $\tilde{y}_{0 r} \prec \tilde{0}$ and there exists a nonbasic index $k \in N$ such that $\tilde{y}_{r k} \prec \tilde{0}$, then pivoting on $\tilde{y}_{r k}$ will yield a dual feasible tableau with a corresponding non-increasing objective value.

Proof. Pivoting on the pivot $\tilde{y}_{r k}$ will result in the new objective row as follows:

$$
\tilde{z}_{j}-\tilde{c}_{j}-\frac{\tilde{y}_{r j}}{\tilde{y}_{r k}}\left(\tilde{z}_{k}-\tilde{c}_{k}\right), j \in N .
$$

For the new tableau to be dual feasible we need to have

$$
\tilde{z}_{j}-\tilde{c}_{j}-\frac{\tilde{y}_{r j}}{\tilde{y}_{r k}}\left(\tilde{z}_{k}-\tilde{c}_{k}\right) \succeq \tilde{0}, j \in N,
$$

which results in

$$
\frac{\tilde{z}_{j}-\tilde{c}_{j}}{\tilde{y}_{r j}} \preceq \frac{\tilde{z}_{k}-\tilde{c}_{k}}{\tilde{y}_{r k}}, \quad \tilde{y}_{r j} \prec \tilde{0} .
$$




\begin{tabular}{cccccccccccc}
\hline Basis & $\ldots$ & $\tilde{x}_{k}$ & $\ldots$ & $\tilde{x}_{j}$ & $\ldots$ & $\tilde{x}_{B_{1}}$ & $\ldots$ & $\tilde{x}_{B_{r}}$ & $\ldots$ & $\tilde{x}_{B_{m}}$ & R.H.S \\
\hline$\tilde{z}$ & $\ldots$ & $\tilde{z}_{k}-\tilde{c}_{k}$ & $\ldots$ & $\tilde{z}_{j}-\tilde{c}_{j}$ & $\ldots$ & $\tilde{0}$ & $\ldots$ & $\tilde{0}$ & $\ldots$ & $\tilde{0}$ & $\tilde{c}_{B} \tilde{B}^{-1} b$ \\
\hline$\tilde{x}_{B_{1}}$ & $\ldots$ & $\tilde{y}_{1 k}$ & $\ldots$ & $\tilde{y}_{1 j}$ & $\ldots$ & $\tilde{1}$ & $\ldots$ & 0 & $\ldots$ & 0 & $\tilde{y}_{01}$ \\
$\vdots$ & $\vdots$ & $\vdots$ & $\vdots$ & $\vdots$ & $\vdots$ & $\vdots$ & $\vdots$ & $\vdots$ & $\vdots$ & $\vdots$ & $\vdots$ \\
$\tilde{x}_{B_{r}}$ & $\ldots$ & $\tilde{y}_{r k}$ & $\ldots$ & $\tilde{y}_{r j}$ & $\ldots$ & $\tilde{0}$ & $\ldots$ & $\tilde{\tilde{1}}$ & $\ldots$ & $\tilde{\tilde{0}}$ & $\tilde{y}_{0 r}$ \\
$\vdots$ & $\vdots$ & $\vdots$ & $\vdots$ & $\vdots$ & $\vdots$ & $\vdots$ & $\vdots$ & $\vdots$ & $\vdots$ & $\vdots$ & $\vdots$ \\
$\tilde{x}_{B_{m}}$ & $\ldots$ & $\tilde{y}_{m k}$ & $\ldots$ & $\tilde{y}_{m j}$ & $\ldots$ & $\tilde{0}$ & $\ldots$ & $\tilde{0}$ & $\ldots$ & $\tilde{1}$ & $\tilde{y}_{0 m}$ \\
\hline
\end{tabular}

Table 1: Tableau of the FFLP problem.

\begin{tabular}{cccccc}
\hline Basis & $\tilde{x}_{1}$ & $\tilde{x}_{2}$ & $\tilde{x}_{3}$ & $\tilde{x}_{4}$ & R.H.S \\
\hline$\tilde{z}$ & $(-2,2,2,2)$ & $(0,2,1,1)$ & $\tilde{0}$ & $\tilde{0}$ & $\tilde{0}$ \\
\hline$\tilde{x}_{3}$ & $(2,4,1,1)$ & $(-3,-1,2,2)$ & $(1,1,0,0)$ & $\tilde{0}$ & $(-4,-4,2,2)$ \\
$\tilde{x}_{4}$ & $(-2,0,1,1)$ & $(-2,4,2,2)$ & $\tilde{0}$ & $(1,1,0,0)$ & $(-1,5,3,3)$ \\
\hline
\end{tabular}

Table 2: First dual feasible simplex tableau.

To satisfy (12), it is sufficient to let

$$
\frac{\tilde{z}_{k}-\tilde{c}_{k}}{\tilde{y}_{r k}} \approx \max \left\{\frac{\tilde{z}_{j}-\tilde{c}_{j}}{\tilde{y}_{r j}} \mid \tilde{y}_{r j} \prec \tilde{0}\right\} .
$$

We note that the new objective value is nonincreasing, since

$$
\tilde{c}_{B} \tilde{B}^{-1} \tilde{b}-\frac{\tilde{y}_{0 r}}{\tilde{y}_{r k}}\left(\tilde{z}_{k}-\tilde{c}_{k}\right) \preceq \tilde{c}_{B} \tilde{B}^{-1} \tilde{b}
$$

based on the fact that

$$
\tilde{y}_{0 r} \prec \tilde{0}, \tilde{y}_{r k} \prec \tilde{0} \text { and } \tilde{z}_{k}-\tilde{c}_{k} \succeq \tilde{0} .
$$

Now, using the above results, we introduce a new dual algorithm to solve the (FFLP) problem directly, making use of the dual feasible simplex tableau. Thus, we refer to the new algorithm as a dual simplex method.

\section{Algorithm: a dual simplex method}

(Dual feasibility) Let $\tilde{B}$ be a basis for the (FFLP) problem such that $\tilde{z}_{j}-\tilde{c}_{j} \succeq \tilde{0}$ for all $j$.

Compute the simplex tableau.

If $\tilde{y}_{0} \succeq \tilde{0}$ then Stop (the current solution is optimal)

else select the pivot row $r$ with $\tilde{y}_{0 r} \prec \tilde{0}$.

If $\tilde{y}_{r j} \succeq \tilde{0}$ for all $j$ then Stop (the primal (FFLP) is infeasible)

else select the pivot column $k$ by means of the following maximum ratio test:

$$
\frac{\tilde{z}_{k}-\tilde{c}_{k}}{\tilde{y}_{r k}} \approx \max \left\{\frac{\tilde{z}_{j}-\tilde{c}_{j}}{\tilde{y}_{r j}} \mid \tilde{y}_{r j} \prec \tilde{0}\right\} .
$$

Pivot on $\tilde{y}_{r k}$.

Remark 29. If $\tilde{A}$ and $\tilde{c}$ are crisp numbers, then our method reduce to dual simplex method in [8]. If
$\tilde{A}$ is a crisp matrix, then the method matches the proposed method in [6]. Moreover, if the all data and variables are crisp numbers, then the method is consistent with the dual simplex method for crisp linear programming.

For an illustration of the dual simplex method we consider the following example.

\section{Example 1.}

$$
\begin{array}{cl}
\max \tilde{z} & \approx(-2,2,2,2) \tilde{x}_{1}+(-2,0,1,1) \tilde{x}_{2} \\
\text { s.t. } & \\
& (2,4,1,1) \tilde{x}_{1}-(1,3,2,2) \tilde{x}_{2}+(1,1,0,0) \tilde{x}_{3} \\
& \approx(-4,-4,2,2), \\
& (-2,0,1,1) \tilde{x}_{1}+(-2,4,2,2) \tilde{x}_{2}+(1,1,0,0) \tilde{x}_{4} \\
& \approx(-1,5,3,3), \\
& \tilde{x}_{1}, \tilde{x}_{2}, \tilde{x}_{3}, \tilde{x}_{4} \succeq \tilde{0} .
\end{array}
$$

We may write the first dual feasible simplex tableau as showed in Table 2.

Since $\tilde{y}_{01} \prec \tilde{0}$, thus $\tilde{x}_{3}$ is a leaving variable and

$$
\max \left\{\frac{\tilde{z}_{j}-\tilde{c}_{j}}{\tilde{y}_{1 j}}: \tilde{y}_{1 j} \prec \tilde{0}\right\} \approx \max \left\{\frac{(0,2,1,1)}{(-3,-1,2,2)}\right\},
$$

thus $\tilde{x}_{2}$ is an entering variable. The new tableau is shown in Table 3.

Therefore, the optimal solution of the (FFLP) problem obtained by the dual method is $\tilde{x}_{1} \approx$ $\tilde{0}, \quad \tilde{x}_{2} \approx\left(\frac{2}{3}, \frac{10}{3}, 10,10\right), \quad \tilde{x}_{3} \approx \tilde{0}$ and $\tilde{x}_{4} \approx$ $\left(-13,13, \frac{269}{3}, \frac{269}{3}\right)$ with the optimal value

$$
\tilde{z} \approx\left(-\frac{16}{3}, \frac{4}{3}, \frac{70}{3}, \frac{70}{3}\right) .
$$

\section{Acknowledgements}

Research partially supported by the projects TIN2011-27696-C02-01 from the Government of Spain and P07-TIC02970 from the Government of Andalucia. 


\begin{tabular}{cccccc}
\hline Basis & $\tilde{x}_{1}$ & $\tilde{x}_{2}$ & $\tilde{x}_{3}$ & $\tilde{x}_{4}$ & R.H.S \\
\hline$\tilde{z}$ & $\left(-\frac{23}{6}, \frac{41}{6}, \frac{97}{6}, \frac{97}{6}\right)$ & $(-2,2,10,10)$ & $\left(-\frac{1}{3}, \frac{4}{3}, \frac{23}{6}, \frac{23}{6}\right)$ & 0 & $\left(-\frac{16}{3}, \frac{4}{3}, \frac{70}{3}, \frac{70}{3}\right)$ \\
\hline$\tilde{x}_{2}$ & $\left(-\frac{19}{6}, \frac{1}{6}, 7,7\right)$ & $(1,1,4,4)$ & $\left(-\frac{5}{6},-\frac{1}{6}, 2,2\right)$ & 0 & $\left(\frac{2}{3}, \frac{10}{3}, 10,10\right)$ \\
$\tilde{x}_{4}$ & $\left(-\frac{29}{3}, \frac{32}{3}, \frac{89}{4}, \frac{89}{4}\right)$ & $(-6,6,20,20)$ & $\left(-\frac{3}{2}, \frac{7}{2}, \frac{23}{3}, \frac{23}{3}\right)$ & $(1,1,0,0)$ & $\left(-13,13, \frac{269}{3}, \frac{269}{3}\right)$ \\
\hline
\end{tabular}

Table 3: New tableau obtained when $\tilde{x}_{3}$ leaves and $\tilde{x}_{2}$ enters.

\section{References}

[1] T. Allahviranloo, F.H. Lotfi, M.K. Kiasary, N.A. Kiani, L. Alizadeh, Solving full fuzzy linear programming problem by the ranking function, Appl. Math. Sci. 2(2008) 19-32.

[2] R.E. Bellman, L.A. Zadeh, Decision making in a fuzzy environment, Management Science 17 (1970) 141-164.

[3] A. Ebrahimnejad, S.H. Nasseri, Linear programs with trapezoidal fuzzy numbers: a duality approach, International Journal of Operational Research (IJOR), 13(1) (2012) 67-89.

[4] K. Ganesan, P. Veeramani, Fuzzy linear programs with trapezoidal fuzzy numbers, Ann. Oper. Res., 143 (2006) 305-315.

[5] F.H. Lotfi, T. Allahviranloo, M.A. Jondabeha, L. Alizadeh, Solving fully fuzzy linear programming using lexicography method and fuzzy approximate solution, Appl.Math. Model., 33(2009) 3151-3156.

[6] B. Kheirfam, J.L. Verdegay, The dual simplex method and sensitivity analysis for fuzzy linear programming with symmetric trapezoidal numbers, Fuzzy Optim. Decis. Making, 12(2013) 171-189.
[7] A. Kumar, J. Kaur, P. Singh, A new method for solving fully fuzzy linear programming problems, Appl. Math. Model., 35(2011) 817-823.

[8] N. Mhdavi-Amiri, S. H. Nasseri, Duality results and a dual simplex method for linear programming problems with trapezoidal fuzzy variables, Fuzzy Sets and Systems, 158 (2007) 1961-1978.

[9] S.H. Nasseri, A. Ebrahimnejad, S. Mizuno, Duality in fuzzy linear programming with symmetric trapezoidal numbers, Applications and Applied Mathematics: An International Journal, 5(10) (2010) 1469-1484.

[10] H. Tanaka, K. Asai, Fuzzy linear programming problems with fuzzy numbers, Fuzzy Sets and Systems 13 (1984) 1-10.

[11] J.L. Verdegay, Fuzzy Mathematical Programming, In "Fuzzy Information and Decision Processes". M.M. Gupta and E. Sanchez (Eds). North Holland (1982), 231-237.

[12] H. J. Zimmermann, Fuzzy programming and linear programming with several objective functions, Fuzzy Set and Systems 1 (1978) 45-55. 